\title{
Determination of melanin types and relative concentrations: an observational study using a non-invasive inverse skin reflectance analysis
}

\begin{abstract}
Objective: Melanin is a major skin colour pigment that made up of eumelanin (the dark brown-black colour) and pheomelanin (the light red-yellow colour) pigments. Skinwhitening products typically contain depigmentation agents that reduce the level of pigmentation by changing the pheomelanin-eumelanin production. Similarly, in skin pigment treatment of skin disorders, the melanin production is managed accordingly. To assess and improve treatment efficacy, it is important to have a measurement tool that is capable of determining the melanin types objectively. So far, the efficacy assessment is subjective. In this study, an inverse skin reflectance pigmentation analysis system that determines eumelanin and pheomelanin content is developed and evaluated in an observational study involving 36 participants with skin photo type IV. Methods: The reflectance spectra of the left forearms of participants were analysed by the pigmentation analysis system to determine their skin parameters - pheomelanin and eumelanin concentrations, melanosome volume fraction, and epidermal thickness. The determined skin parameters are then inputted into the realistic skin model (RSM) of the Advanced Systems Analyses Program (asap®) to generate the ground truth reflectance spectra for the given skin parameters to validate the system. Results: The developed pigmentation analysis system is found to be accurate with a spectral error of $0.0163 \pm 0.009$ between measured reflectance and the reflectance output of the analysis system and RSM. The regression analysis shows a strong linear relationship (R2 = $0.994)$ indicating good precision. The relative concentrations of pheomelanin $(38.23 \pm 15.04)$ and eumelanin $(1.68 \pm 0.91)$ analysed by the system gives a ratio of pheomelanin to eumelanin of $0.048 \pm 0.029$; this value is consistent with previously reported figure of 0.049 . Conclusion: The proposed pigmentation analysis system is able to determine melanin types and their relative concentrations. It has the potential to assess the efficacy of the skinwhitening and pigmentation treatments objectively in a non-invasive manner.
\end{abstract}

Keyword: Determination; Melanin types; Non-invasive; Pigmentation analysis system; Skin reflectance 